\title{
Serum B7-H4 expression is a significant prognostic indicator for patients with gastric cancer
}

\author{
Hongbing Shi ${ }^{1}$, Mei $\mathrm{Ji}^{1}$, Jun $\mathrm{Wu}^{1}$, Qi Zhou ${ }^{1}$, Xiaodong $\mathrm{Li}^{1}$, Zhengguang $\mathrm{Li}^{1}$, Xiao Zheng ${ }^{2}$, Bing X X ${ }^{2}$, Weiqing Zhao ${ }^{1}$,
} Changping $\mathrm{Wu}^{1,2^{*}}$ and Jingting Jiang ${ }^{1,2^{*}}$

\begin{abstract}
Background: B7-H4 is a novel B7 ligand that plays an important role in the T cell-mediated immune response as a negative regulator. Previous studies have suggested the aberrant expression of membrane B7-H4 in tumor cells. The aim of this study is to determine the expression levels of preoperative soluble $\mathrm{B} 7-\mathrm{H} 4$ (sB7-H4) in circulation and to investigate the correlations between sB7-H4 levels and clinicopathological parameters as well as the survival rate of patients with gastric cancer.
\end{abstract}

Methods: Blood specimens from 132 patients with gastric cancer and 63 healthy volunteers were analyzed by sandwich enzyme-linked immunosorbent assay.

Results: Median concentrations of sB7-H4 in patients with gastric cancer were significantly higher than those in healthy volunteers (16.85 versus $10.46 \mathrm{ng} / \mathrm{mL} ; P=0.008$ ). Median levels of sB7-H4 were significantly correlated with tumor size, lymph node metastasis, the depth of tumor invasion and tumor-node-metastasis classification $(P=0.002$, $P=0.001, P=0.041$ and $P<0.001$, respectively), but not with sex, age, tumor location or histological subtype (all $P>0.05)$. Additionally, the overall survival rate was significantly lower in patients with high sB7-H4 levels when compared with low sB7-H4 levels (50.0\% versus $\left.77.3 \%, X^{2}=10.78, P=0.001\right)$. Moreover, multivariate analysis demonstrated that the risk of death was significantly higher in patients with high sB7-H4 levels than in those with low sB7-H4 levels $(P=0.039)$.

Conclusions: $\mathrm{SB} 7-\mathrm{H} 4$ is a valuable blood marker for predicting the progression and prognosis of patients with gastric cancer.

Keywords: Gastric cancer, sB7-H4, Prognosis, ELISA

\section{Background}

Gastric cancer is one of the most common types of cancer worldwide in terms of incidence and mortality [1], especially in China [2]. Although multi-model treatment strategies including surgery, perioperative chemotherapy, radiotherapy and immunotherapy are used, the five-year survival rate for patients suffering from gastric cancer is still $25 \%$ or less [3-7]. Therefore, it is necessary to improve current therapeutic modalities and to explore new biological molecular markers for predicting the progression of gastric cancer and helping targeted therapy.

\footnotetext{
*Correspondence: czsdyrmyyzlk@163.com; jjtnew@163.com

'Department of Oncology, The Third Affiliated Hospital of Soochow University, 185 Juqian Street, Changzhou 213003, Jiangsu Province, People's Republic of China

${ }^{2}$ Department of Tumor Biological Treatment, The Third Affiliated Hospital of Soochow University, 185 Juqian Street, Changzhou 213003, Jiangsu Province, People's Republic of China
}

Recently, experimental evidence has indicated that B7 family molecules may participate in the positive and negative regulation of cell-mediated immunity in peripheral tissues [8]. Recent findings have demonstrated that B7-H1 (PD-L1), B7-H2 (PD-L2), B7-H3 and B7-H4 are aberrantly expressed in some tumor tissues and/or sera of cancer patients, suggesting that these molecules might be new molecular biomarkers for tumor diagnosis and prognosis [9-11]. B7-H4 has been identified through the National Center for Biotechnology Information (NCBI) database searching and cDNA library screening to reveal that its sequence contains B7 extracellular immunoglobulin domains [12-14]. Previous studies have showed that $\mathrm{B} 7-\mathrm{H} 4$ can regulate $\mathrm{T}$ cell-mediated immune response through inhibiting $\mathrm{T}$ cell proliferation, cytokine 
secretion and the development of cytotoxicity [15-19]. It has been reported that $\mathrm{B} 7-\mathrm{H} 4$ is expressed at high levels in many cancer tissues such as breast, ovarian, lung, pancreatic, renal cell and gastric cancers [10,20-27]. Simon et al. reported that B7-H4 is elevated in serum samples from ovarian cancer patients when compared with healthy controls or women with benign gynecological diseases [10]. However, the clinical significance of B7-H4 expression in blood specimens from gastric cancer patients has not yet been determined.

In this study, we examined circulating B7-H4 levels in blood specimens from patients with gastric cancer using an sandwich enzyme-linked immunosorbent assay (ELISA) kit for soluble B7-H4 (sB7-H4). Additionally, we investigated the correlation between sB7-H4 levels and clinicopathological parameters, and patient survival rate. Our results showed that the evaluation of sB7-H4 levels could help for predicting the progression and prognosis of patients with gastric cancer.

Table 1 Correlation between sB7-H4 levels and clinical characteristics of patients

\begin{tabular}{|c|c|c|c|c|}
\hline Characteristics & $N$ & Median (range) & $\mathbf{Z}$ & $P$ \\
\hline$\overline{\text { Sex }}$ & & & 0.936 & 0.349 \\
\hline Male & 97 & $15.76(0.11-182.58)$ & & \\
\hline Female & 35 & $21.28(1.86-171.31)$ & & \\
\hline Age(years) & & & 0.691 & 0.489 \\
\hline$\geq 60$ & 75 & $15.21(0.11-182.58)$ & & \\
\hline$<60$ & 57 & $18.28(2.59-170.77)$ & & \\
\hline Tumor location & & & 1.363 & 0.506 \\
\hline Gastric cardia & 21 & $25.26(2.47-121.96)$ & & \\
\hline Gastric body & 54 & $14.97(1.35-182.58)$ & & \\
\hline Gastric antrum & 57 & $17.82(0.11-158.60)$ & & \\
\hline Tumor size & & & 3.12 & 0.002 \\
\hline$<5 \mathrm{~cm}$ & 85 & $13.96(0.11-158.60)$ & & \\
\hline$\geq 5 \mathrm{~cm}$ & 47 & $28.47(1.35-182.58)$ & & \\
\hline Lymph node metastasis & & & 3.392 & 0.001 \\
\hline Negative & 50 & $10.92(0.11-144.97)$ & & \\
\hline Positive & 82 & $21.32(1.35-182.58)$ & & \\
\hline Depth of tumor invasion & & & 2.039 & 0.041 \\
\hline pT1-T2 & 35 & $12.22(1.51-109.66)$ & & \\
\hline pT3-T4 & 97 & $19.65(0.11-182.58)$ & & \\
\hline Histology differentiation & & & 1.045 & 0.296 \\
\hline Differentiated & 67 & $18.28(1.35-171.31)$ & & \\
\hline Poorly differentiated & 65 & $15.16(0.11-182.58)$ & & \\
\hline Stage & & & 3.524 & $<0.001$ \\
\hline$|+| \mid$ & 69 & $12.22(0.11-144.97)$ & & \\
\hline$I I I+I V$ & 63 & $22.72(1.35-182.58)$ & & \\
\hline
\end{tabular}

\section{Methods}

\section{Selection of patients}

Blood specimens were preoperatively collected from 132 primary gastric cancer patients (97 men and 35 women; age range 30 to 86-years-old; average age 61.39-yearsold) treated surgically at the Third Affiliated Hospital of Soochow University (Jiangsu Province, China) between 2008 and 2010. Patients who had undergone any form of preoperative chemotherapy and/or radiation therapy were excluded. Furthermore, none of patients enrolled in this study suffered from other cancers. Each patient with gastric cancer was classified on the basis of the tumornode-metastasis (TNM) classification of the International Union against Cancer (UICC) [28]. Peripheral blood specimens from 63 healthy volunteers (39 men and 24 women; age range 25 to 87 -years old; average age 48.91 -years-old) who had never received a diagnosis of malignancy were chosen as the control group. The remaining clinical and pathological features are shown in Table 1. Collected samples were kept at room temperature (RT) for a minimum of 30 minutes (and a maximum of 60 minutes), and serum was obtained after centrifugation at $4000 \mathrm{rpm}$ at $4^{\circ} \mathrm{C}$ for 10 minutes. The serum was immediately removed and frozen on dry ice at $-80^{\circ} \mathrm{C}$ until use.

Before enrollment this study protocol was approved by the ethics committee of Soochow University and this study was conducted in accordance with the principles of the Declaration of Helsinki and Good Clinical Practice Guidelines. Patients and healthy volunteers provided written informed consent for all specimens collected.

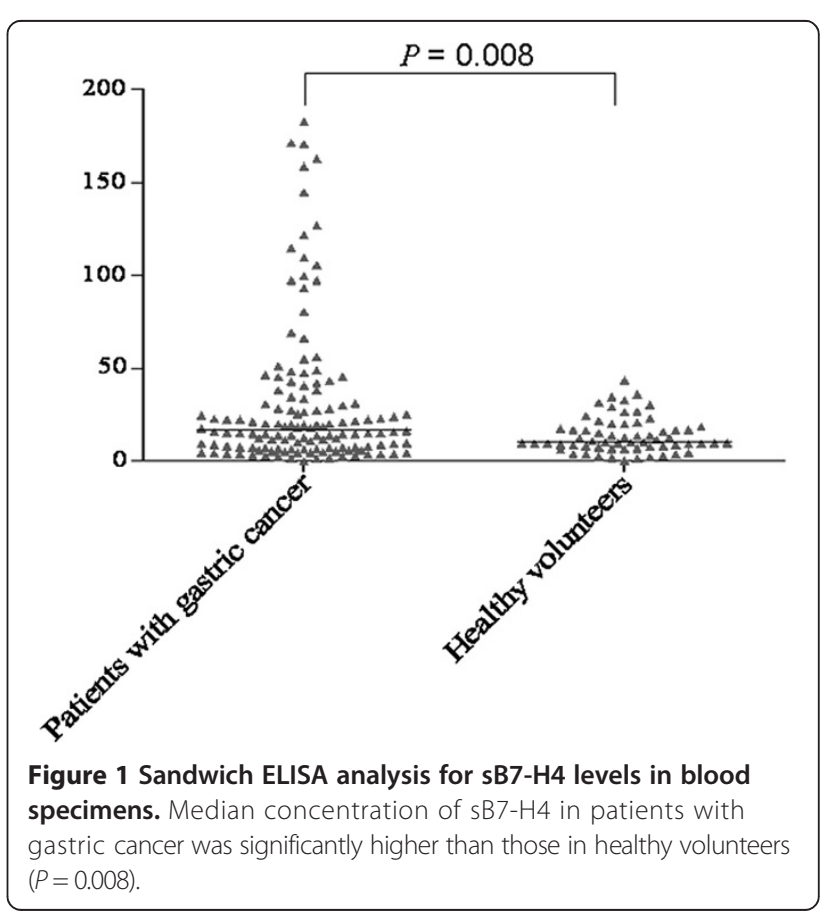




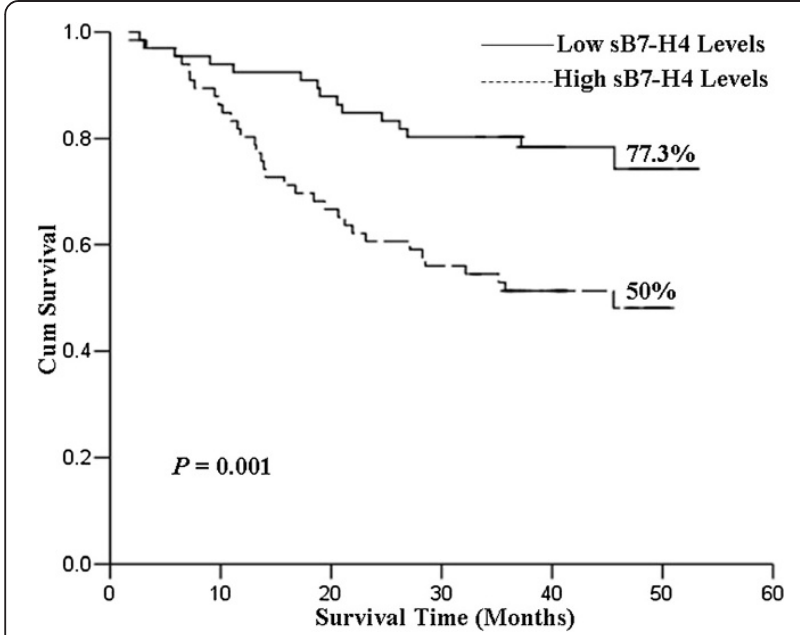

Figure 2 All patients were divided into low $(n=66)$ and high $(n=66)$ sB7-H4 level groups. Survival curves were analyzed by Kaplan-Meier method and log-rank test. Patients with high sB7-H4 levels had a significantly poorer survival rate when compared to those with low sB7-H4 levels $(P=0.001)$

\section{Sandwich ELISA detection for sB7-H4}

Simon et al. have developed a sensitive sandwich ELISA to analyze the expression level of sB7-H4 in serum samples from patients with ovarian cancer [10]. A similar protocol was used for the blood specimens from patients with gastric cancer and the healthy volunteers. Briefly, $25 \mu \mathrm{L}$ of the undiluted blood specimen was added to high-binding polystyrene plates coated with capture mAb Clone H74 (eBioscience, San Diego, United States). Immobilized antigen was detected with diluted biotinylated secondary mAb (eBioscience, San Diego, United States) followed by horseradish peroxidase-conjugated streptavidin (Biolegend Inc., Californian, United States). For calibration, the standards of recombinant protein and two controls were conducted in parallel with the test samples on each plate.

\section{Statistical analysis}

Due to non-normal distribution, differences between the median groups were evaluated by the Mann-Whitney U test. Survival time was calculated from the first day of diagnosis to the date of last follow-up or death, and the median follow-up period after diagnosis was 35 months (range, 1 to 61 months). Survival curves were analyzed using Kaplan-Meier curves and differences in survival rates were examined using the log-rank test. Univariate and multivariate analyses (Cox proportional hazards regression model) were performed to evaluate the prognosis factors for gastric cancer. All statistical analyses were performed using the Statistical Package for the Social Sciences, version 13.0 (SPSS, Chicago, Illinois, United States). A statistically significant difference was considered to be a $P$ value less than 0.05 .

\section{Results}

\section{Analysis of sB7-H4 in gastric cancer patients versus} healthy controls

Sandwich ELISA was used to assay the levels of sB7-H4 in serum samples from 132 patients with gastric cancer and 63 healthy volunteers. As shown in Figure 1, an elevated level (median) of sB7-H4 in serum samples from patients with gastric cancer $(16.85 \mathrm{ng} / \mathrm{mL}$ with a range of 0.11 to $182.58 \mathrm{ng} / \mathrm{mL}$ ) was observed when compared with that from healthy volunteers $(10.46 \mathrm{ng} / \mathrm{mL}$ with a range of 0.47 to $43.23 \mathrm{ng} / \mathrm{mL} P=0.008$ ).

\section{Relationship between sB7-H4 expression and clinicopathological factors in patients with gastric cancer} With the extension of tumors, the sB7-H4 levels tended to increase in blood specimens from gastric cancer patients. As shown in Table 1, the median sB7-H4 level was significantly higher in gastric cancer patients with tumor size of more than or equal to $5 \mathrm{~cm}$ than in patients with tumor size of less than $5 \mathrm{~cm}(P=0.002)$. Patients with lymph node metastasis had higher sB7-H4 levels when compared with those without lymph node metastasis $(P=0.001)$. In addition, sB7-H4 levels revealed an enhancement with the depth increase of tumor invasion and TNM stage $(P=0.041$ and $P<0.001$, respectively). However, no statistically significant correlation between

Table 2 Univariate and multivariate analyses of overall survival in patients

\begin{tabular}{|c|c|c|c|c|c|}
\hline \multirow{2}{*}{$\begin{array}{l}\text { Clinicopathological } \\
\text { parameters }\end{array}$} & \multirow{2}{*}{$\begin{array}{l}\text { Comparison/ } \\
\text { reference }\end{array}$} & \multicolumn{2}{|c|}{ Univariate analysis } & \multicolumn{2}{|c|}{ Multivariate analysis } \\
\hline & & Hazard ratio $(95 \% \mathrm{Cl})$ & $P$ value & Hazard ratio $(95 \% \mathrm{Cl})$ & $P$ value \\
\hline sB7-H4 expression & High/Low & $2.677(1.452-4.934)$ & 0.002 & $1.925(1.033-3.857)$ & 0.039 \\
\hline Gender & Male/Female & $1.153(0.618-2.148)$ & 0.655 & $1.194(0.772-1.873)$ & 0.497 \\
\hline Age & $<60 / \geq 60$ & $0.988(0.962-1.015)$ & 0.393 & $1.014(0.529-1.384)$ & 0.859 \\
\hline Tumor size & $<5 \mathrm{~cm} / \geq 5 \mathrm{~cm}$ & $2.714(1.536-4.794)$ & 0.001 & $1.008(0.284-1.637)$ & 0.359 \\
\hline Lymph node metastasis & Positive/Negative & $5.792(2.458-13.644)$ & $<0.001$ & $1.623(0.633-2.365)$ & 0.195 \\
\hline Depth of tumor invasion & pT3-T4/pT1-T2 & $2.714(1.536-4.794)$ & 0.001 & $1.842(0.825-1.972)$ & 0.156 \\
\hline TNM stage & $\|I I, I V /\|$, & $5.966(2.962-12.013)$ & $<0.001$ & $5.184(2.544-10.563)$ & $<0.001$ \\
\hline
\end{tabular}


sB7-H4 level and sex, age, tumor location or histological subtype was observed $(P=0.349, P=0.489, P=0.506$ and $P=0.296$, respectively).

\section{Relationship between sB7-H4 expression and prognosis} Based on the median value of sB7-H4 levels, we used $16.85 \mathrm{ng} / \mathrm{mL}$ as the cutoff value to divide all patients into groups with low $(\mathrm{n}=66)$ and high $(\mathrm{n}=66)$ sB7-H4 levels. The overall survival rates of patients with low and high levels of $\mathrm{B} 7-\mathrm{H} 4$ were 77.3 and $50.0 \%$, respectively (Figure 2) $\left(X^{2}=10.78, P=0.001\right)$. As shown in Table 2, univariate analysis showed that tumor size, lymph node metastasis, depth of tumor invasion, TNM stage and sB7-H4 expression were significantly related to overall survival $(P=0.001, P<0.001, P=0.001, P<0.001$ and $P=0.002$, respectively). Multivariate analysis indicated that the death risk of gastric cancer patients in the high B7-H4 level group was significantly higher $(P=0.039)$. As expected, the higher TNM stage was also significantly associated with an elevated risk of death for gastric patients $(P<0.001)$.

\section{Discussion}

B7-H4 is a member of B7 family which inhibits tumorspecific T cell-mediated immune response [29]. Previous studies have showed that the expression levels of B7-H4 were significantly higher in many cancerous cells of gastric cancer tissues than that in the gastric polyp tissues or adjacent normal tissues [19]. In the present study, we quantitatively measured the expression levels of sB7-H4 in serum samples from patients with gastric cancer and healthy volunteers by sandwich ELISA. Compared with the level of sB7-H4 in healthy volunteers, sB7-H4 level was significantly increased in patients with gastric cancer. Additionally, sB7-H4 was detected in blood samples from patients with renal cell carcinoma and ovarian cancer according to ELISA assays [10,30]. Therefore, sB7-H4 is not specific to gastric cancer and might serve as a potential serum biomarker of various malignancies.

We have found that sB7-H4 is significantly correlated with tumor size, lymph node metastasis, depth of tumor invasion and TNM stage, indicating that sB7-H4 may be a valuable marker for predicting tumor progression in patients with gastric cancer. In fact, Arigami et al. [31] have found that B7-H4 mRNA copies in patients with gastric cancer are significantly correlated with the depth of tumor invasion, lymph node metastasis and overall stage through quantitative reverse transcription polymerase chain reaction (RT-PCR) analysis. In addition, the five-year survival rate of $\mathrm{B} 7-\mathrm{H} 4$-positive patients was lower than that of B7-H4-negative patients [31]. The present study exhibited a correlation between the sB7-H4 level and survival rate of patients with gastric cancer. Moreover, the multivariate analysis confirmed that sB7-H4 was an independent factor for affecting the survival time of gastric cancer patients. These results indicated that the assessment of sB7-H4 levels in blood might help for predicting the prognosis of patients with gastric cancer and establishing treatment strategies.

B7-H4 may contribute to the immune system during tumor progression. Some reports have demonstrated that B7-H4 can inhibit CD4 and CD8-positive T lymphocyte proliferation, cell-cycle progression, the production of interleukin (IL)-2, IL-4 and IL-10 and antitumor immunity [12-14]. The overexpression of sB7-H4 in blood from gastric cancer patients may promote tumor progression by providing the mechanism for cancer cells to avoid immune attack. Our study suggests that the blockade of B7-H4 may be beneficial for the enhancement of immunological function and the prognosis of gastric cancer patients.

\section{Conclusions}

We have demonstrated that $\mathrm{sB} 7-\mathrm{H} 4$ is a promising serum biomarker that may help to improve progression and prognostic assessment of gastric cancer. Furthermore, B7-H4 inhibition may be a useful method for treating gastric cancer. However, further studies are needed to explore the potential role of monitoring cancer cells in patients after surgery and chemotherapy.

\section{Abbreviations}

ELISA: Sandwich enzyme-linked immunosorbent assay; TNM: Tumor-nodemetastasis; NCBI: National Center for Biotechnology Information; RT-PCR: Reverse transcription polymerase chain reaction.

\section{Competing interests}

The authors declare that they have no competing interests.

\section{Authors' contributions}

CW and JJ conceived and designed the experiments. HS, QZ and WZ performed the experiments. XZ and BX analyzed the data. HS, ZL and XL wrote the paper. MJ and JW edited the manuscript. All authors read and approved the final manuscript.

\section{Acknowledgements}

This research project was supported by the National Natural Science Foundation of China (NSFC) (81171653 and 30972703) and Natural Science Foundation of Jiangsu Province (BK2011246 and BK2011247).

Received: 22 October 2013 Accepted: 8 June 2014 Published: 19 June 2014

\section{References}

1. Hartgrink HH, Jansen EP, van Grieken NC, van de Velde CJ: Gastric cancer. Lancet 2009, 374:477-490.

2. Yeh JM, Kuntz KM, Ezzati M, Goldie SJ: Exploring the cost-effectiveness of Helicobacter pylori screening to prevent gastric cancer in China in anticipation of clinical trial results. Int J Cancer 2009, 124:157-166.

3. Bouche O, Ychou M, Burtin P, Bedenne L, Ducreux M, Lebreton G, Baulieux J, Nordlinger B, Martin C, Seitz JF, Tigaud JM, Echinard E, Stremsdoerfer N, Milan C, Rougier P: Adjuvant chemotherapy with 5-fluorouracil and cisplatin compared with surgery alone for gastric cancer: 7-year results of the FFCD randomized phase III trial (8801). Ann Oncol 2005, 16:1488-1497.

4. Cunningham D, Allum WH, Stenning SP, Thompson JN, Van de Velde CJ, Nicolson M, Scarffe JH, Lofts FJ, Falk SJ, Iveson TJ, Smith DB, Langley RE, Verma M, Weeden S, Chua YJ, Participants MT: Perioperative 
chemotherapy versus surgery alone for resectable gastroesophageal cancer. N Engl J Med 2006, 355:11-20.

5. Macdonald JS, Smalley SR, Benedetti J, Hundahl SA, Estes NC, Stemmermann GN, Haller DG, Ajani JA, Gunderson LL, Jessup JM, Martenson JA: Chemoradiotherapy after surgery compared with surgery alone for adenocarcinoma of the stomach or gastroesophageal junction. N Engl J Med 2001, 345:725-730.

6. Nashimoto A, Nakajima T, Furukawa H, Kitamura M, Kinoshita T, Yamamura Y, Sasako M, Kunii Y, Motohashi H, Yamamoto S: Randomized trial of adjuvant chemotherapy with mitomycin, Fluorouracil, and Cytosine arabinoside followed by oral Fluorouracil in serosa-negative gastric cancer: Japan Clinical Oncology Group 9206-1. J Clin Oncol 2003, 21:2282-2287.

7. Orditura M, De Vita F, Muto P, Vitiello F, Murino P, Lieto E, Vecchione L, Romano A, Martinelli E, Renda A, Ferraraccio F, Del Genio A, Ciardiello F, Galizia G: Adjuvant chemoradiotherapy in patients with stage III or IV radically resected gastric cancer: a pilot study. Arch Surg 2010, 145:233-238.

8. Greenwald RJ, Freeman GJ, Sharpe AH: The B7 family revisited. Annu Rev Immunol 2005, 23:515-548.

9. Konishi J, Yamazaki K, Azuma M, Kinoshita I, Dosaka-Akita H, Nishimura M: B7-H1 expression on non-small cell lung cancer cells and its relationship with tumor-infiltrating lymphocytes and their PD-1 expression. Clin Cancer Res 2004, 10:5094-5100.

10. Simon I, Zhuo S, Corral L, Diamandis EP, Sarno MJ, Wolfert RL, Kim NW: B7-h4 is a novel membrane-bound protein and a candidate serum and tissue biomarker for ovarian cancer. Cancer Res 2006, 66:1570-1575.

11. Zang X, Thompson RH, Al-Ahmadie HA, Serio AM, Reuter VE, Eastham JA, Scardino PT, Sharma P, Allison JP: B7-H3 and B7x are highly expressed in human prostate cancer and associated with disease spread and poor outcome. Proc Natl Acad Sci U S A 2007, 104:19458-19463.

12. Prasad DV, Richards S, Mai XM, Dong C: B7S1, a novel B7 family member that negatively regulates T cell activation. Immunity 2003, 18:863-873.

13. Sica GL, Choi IH, Zhu G, Tamada K, Wang SD, Tamura H, Chapoval Al, Flies $\mathrm{DB}$, Bajorath J, Chen L: B7-H4, a molecule of the B7 family, negatively regulates T cell immunity. Immunity 2003, 18:849-861.

14. Zang X, Loke P, Kim J, Murphy K, Waitz R, Allison JP: B7x: a widely expressed B7 family member that inhibits $\mathrm{T}$ cell activation. Proc Natl Acad Sci U S A 2003, 100:10388-10392.

15. Aunoble B, Sanches R, Didier E, Bignon YJ: Major oncogenes and tumor suppressor genes involved in epithelial ovarian cancer (review). Int J Oncol 2000, 16:567-576.

16. Carreno BM, Collins M: BTLA: a new inhibitory receptor with a B7-like ligand. Trends Immunol 2003, 24:524-527.

17. Nicosia SV, Bai W, Cheng JQ, Coppola D, Kruk PA: Oncogenic pathways implicated in ovarian epithelial cancer. Hematol Oncol Clin North Am 2003, 17:927-943.

18. Wang S, Chen L: Co-signaling molecules of the B7-CD28 family in positive and negative regulation of T lymphocyte responses. Microbes Infect 2004, 6:759-766.

19. Zou W, Chen L: Inhibitory B7-family molecules in the tumour microenvironment. Nat Rev Immunol 2008, 8:467-477.

20. Awadallah NS, Shroyer KR, Langer DA, Torkko KC, Chen YK, Bentz JS, Papkoff J, Liu W, Nash SR, Shah RJ: Detection of B7-H4 and p53 in pancreatic cancer: potential role as a cytological diagnostic adjunct. Pancreas 2008, 36:200-206

21. Jiang J, Zhu Y, Wu C, Shen Y, Wei W, Chen L, Zheng X, Sun J, Lu B, Zhang $\mathrm{X}$ : Tumor expression of $\mathrm{B} 7-\mathrm{H} 4$ predicts poor survival of patients suffering from gastric cancer. Cancer Immunol Immunother 2010, 59:1707-1714.

22. Krambeck AE, Thompson RH, Dong H, Lohse CM, Park ES, Kuntz SM, Leibovich BC, Blute ML, Cheville JC, Kwon ED: B7-H4 expression in renal cell carcinoma and tumor vasculature: associations with cancer progression and survival. Proc Natl Acad Sci U S A 2006, 103:10391-10396.

23. Kryczek I, Wei S, Zhu G, Myers L, Mottram P, Cheng P, Chen L, Coukos G, Zou W: Relationship between B7-H4, regulatory T cells, and patient outcome in human ovarian carcinoma. Cancer Res 2007, 67:8900-8905.

24. Simon I, Katsaros D, Rigault de la Longrais I, Massobrio M, Scorilas A, Kim NW, Sarno MJ, Wolfert RL, Diamandis EP: B7-H4 is over-expressed in early-stage ovarian cancer and is independent of CA125 expression. Gynecol Oncol 2007, 106:334-341.

25. Sun Y, Wang Y, Zhao J, Gu M, Giscombe R, Lefvert AK, Wang X: B7-H3 and B7-H4 expression in non-small-cell lung cancer. Lung Cancer 2006 , 53:143-151.
26. Tringler B, Zhuo S, Pilkington G, Torkko KC, Singh M, Lucia MS, Heinz DE, Papkoff J, Shroyer KR: B7-h4 is highly expressed in ductal and lobular breast cancer. Clin Cancer Res 2005, 11:1842-1848.

27. Zheng Y, Katsaros D, Shan SJ, de la Longrais IR, Porpiglia M, Scorilas A, Kim NW, Wolfert RL, Simon I, Li L, Feng Z, Diamandis EP: A multiparametric panel for ovarian cancer diagnosis, prognosis, and response to chemotherapy. Clin Cancer Res 2007, 13:6984-6992.

28. Sobin LH: Wittekind CH International Union Against Cancer: TNM Classification of Malignant Tumors. 6th edition. New York: Wiley-Liss; 2002.

29. Podojil JR, Miller SD: Targeting the B7 family of co-stimulatory molecules: successes and challenges. BioDrugs 2013, 27:1-13.

30. Thompson RH, Zang X, Lohse CM, Leibovich BC, Slovin SF, Reuter VE, Cheville JC, Blute ML, Russo P, Kwon ED, Allison JP: Serum-soluble B7x is elevated in renal cell carcinoma patients and is associated with advanced stage. Cancer Res 2008, 68:6054-6058.

31. Arigami T, Uenosono Y, Hirata M, Hagihara T, Yanagita S, Ishigami S, Natsugoe $\mathrm{S}$ : Expression of B7-H4 in blood of patients with gastric cancer predicts tumor progression and prognosis. J Surg Oncol 2010, 102:748-752.

doi:10.1186/1477-7819-12-188

Cite this article as: Shi et al:: Serum B7-H4 expression is a significant prognostic indicator for patients with gastric cancer. World Journal of Surgical Oncology 2014 12:188.

\section{Submit your next manuscript to BioMed Central and take full advantage of:}

- Convenient online submission

- Thorough peer review

- No space constraints or color figure charges

- Immediate publication on acceptance

- Inclusion in PubMed, CAS, Scopus and Google Scholar

- Research which is freely available for redistribution 